\title{
Effect of Tinnitus on Sound Localizations : Level IV Case-Control Study
}

\author{
Original \\ Article \\ Mayada Elsherif, Meran Samir Allam, Hesham Kozou \\ Department of Otorhinolaryngology, Faculty of Medicine, Alexandria University, Alexandria, \\ Egypt
}

\begin{abstract}
Background: Tinnitus is a prevalent complaint in otorhinolaryngology clinics. Its pathophysiology is multifactorial. The effect of tinnitus on sound localization is still unclear, which is essential for safety.

Objective: To determine the effect of tinnitus on the ability to localize different sound stimuli with or without noise. As we compared localization error scores of tinnitus patients with those of matched controls.

Patients and Methods: Two groups of subjects were studied, a tinnitus group $(n=20)$ and a control group ( $n=20)$. Tinnitus group performed pitch and loudness matches to either an external stimulus.All tinnitus subjects showed bilateral normal hearing up to $4000 \mathrm{~Hz}$. all subjects underwent basic audiological evaluation using pure tone, speech audiometry. Tinnitus group performed pitch and loudness matches to either an external stimulus. Finally, all subjects underwent a sound localization test. The later was performed using six speakers to test the center of the frontal field in favorable and unfavorable conditions. Subject was instructed to point to the direction of sound every time. An error score was calculated based on the difference between the subject's perceived sound speaker and the stimulating speaker.

Results: For both groups, there were significant differences between the total error scores for the different stimuli. The worst sound localization ability was for the $4000 \mathrm{~Hz}$, followed by $250 \mathrm{~Hz}$ and the better ability was for the speech stimuli. Conclusion: The participants with tinnitus exhibited diminished sound localization ability compared with sex-matched controls, and their performance worsened for speech localization in noisy conditions. Further studies with larger sample size across multiple demographic traits are necessary to confirm results.
\end{abstract}

Key Words: Hearing loss, sound localization, tinnitus.

Received: 11 August 2021, Accepted: 05 October 2021

Corresponding Author: Meran Allam, Department of Otorhinolaryngology, Alexandria University, Alexandria Egypt, Tel.: 01156961842, E-mail: samirallam225@gmail.com.

ISSN: 2090-0740, 2021

\section{INTRODUCTION}

Tinnitus, a prevalent complaint in otorhinolaryngology clinics $^{[1]}$ is usually associated with hearing loss. However, to a lesser extent, this disorder can also affect individuals with normal hearing ${ }^{[2]}$. Tinnitus is the phantom perception of a sound in the absence of an external auditory stimulus ${ }^{[3]}$. In some cases, tinnitus can be incapacitating, affecting activities of daily living related to social interaction and sleep, as well as increasing the psychological burden of the patient ${ }^{[4]}$. The pathophysiology of subjective tinnitus is multifactorial, involving both auditory and non-auditory systems ${ }^{[5]}$.

Tinnitus can impair hearing even in those with objectively normal hearing. Furthermore, tinnitus can cause a number of changes in the central auditory system that can lead to impaired temporal resolution ${ }^{[6]}$. Tinnitus can also influence memory-related task performance, attention, and cognitive function ${ }^{[7]}$. However, it is unclear to what extent tinnitus affects sound localization, which is necessary for patient's safety in daily life $\mathrm{e}^{[8]}$.

The objective of the current study was to determine the effect of tinnitus on the ability to localize different sound stimuli with or without background noise. To this end, we compared localization error scores between tinnitus patients and matched controls.

\section{PATIENTS AND METHODS}

This study was conducted in the audio-vestibular unit of the corresponding author's institution during the year of 2020. Approval was obtained from the Ethics Committee of the Faculty of Medicine (IRB NO:00012098). All subjects provided written informed consent prior to participation.

\section{Subjects}

Two groups of subjects were studied, including a tinnitus group $(n=20)$ and a control group $(n=20)$. 
Both groups underwent pure-tone audiometry, and the tinnitus group was selected based on pure-tone audiometry results. All subjects with tinnitus showed bilateral normal hearing up to $4000 \mathrm{~Hz}$. Patients with pulsatile tinnitus, tinnitus for less than three months were excluded. Patients with auditory processing disorder were excluded from the study.

The control group consisted of subjects with no history of tinnitus. Subjects with other neuro-otological complaints were excluded from this study. All subjects underwent an otological examination that showed normal results. Furthermore, no subjects had a history of any other neurological symptoms.

\section{Testing methods}

All subjects responded to the Arabic version of the Dizziness Handicap Inventory ${ }^{[9]}$. Further, all subjects underwent a basic audiological evaluation using puretone and speech audiometry using the commercial system Interacoustics AD629 Diagnostic Audiometer (Interacoustics, Denmark). Both tympanometry and acoustic reflex threshold testing were done using 226 $\mathrm{Hz}$ probe tone were done with Clarinet Clinical Middle Ear Analyzer (Inventis, Italy). The tinnitus group also performed pitch and loudness matches to external stimuli. Finally, all subjects underwent a sound-localization test.

\section{Tinnitus pitch and loudness matching:}

Tinnitus pitch and loudness matching were performed for tinnitus group in a sound-treated room using AD629 Diagnostic Audiometer (Interacoustics, Denmark).

The patients were instructed that the aim of the test is to determine the pitch of their tinnitus. Two stimuli pure tone and narrow band noise were presented and they were asked to tell which one is close to their tinnitus. Pitch matching was done at $1 \mathrm{kHz}$ and the determined level used as the stimulus level to complete pitch matching.

The test started at $1 \mathrm{kHz}$ with one octave steps then inter-octaves were also used for closer approximation, till we obtained the closest tinnitus pitch match and the procedure was repeated 3 times.

Then, the patients were instructed that the purpose of the test was to estimate the loudness of their tinnitus. The stimulus was presented at the patient's threshold level then gradually increased in $5 \mathrm{~dB}$ steps until the loudness matching was obtained. Procedure repeated 3 times to ensure reliability

\section{Sound-localization test setting}

This test was performed in a soundproof room with a 3-m length and 2.50-m width. Adobe Audition was used as the sound creation program, with five speakers used to create the sound stimulus and a separate speaker used to create background noise. We assessed the central quarter of the frontal field. Speakers were positioned in a semicircle at 22.5-degree intervals at $1 \mathrm{~m}$ from the subject (Figure 1:Sound localization Test setting;Speakers were positioned in a semicircle at 22.5-degree intervals 1 $\mathrm{m}$ from the subject.). Subjects were instructed to look at the centered speaker and they were asked to identify the stimulus-presenting speaker by pointing in the perceived direction of the sound under both quiet and background noise conditions. The noise was first directed toward the right ear, and the test was then repeated with the noise directed toward the left ear.

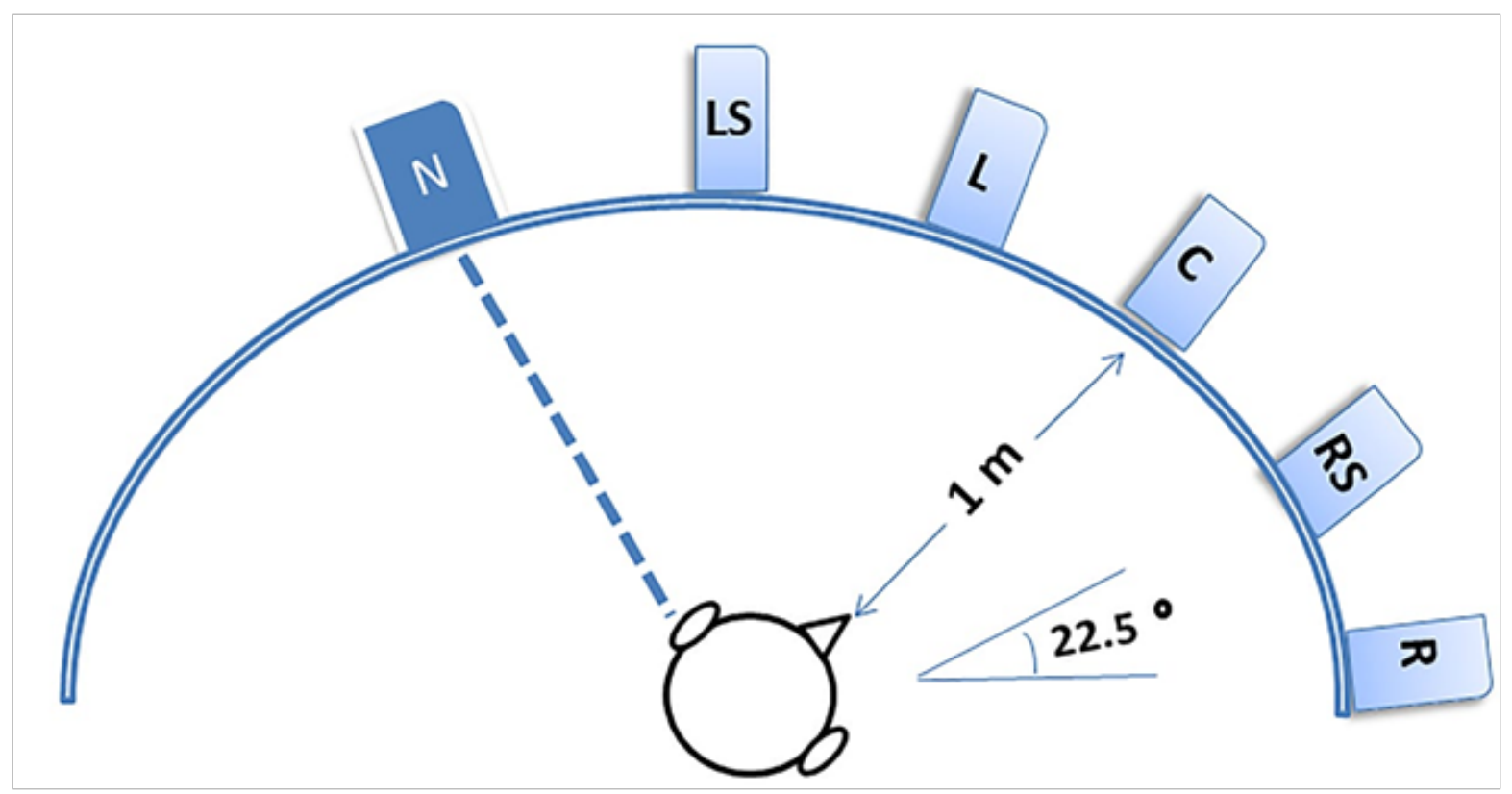




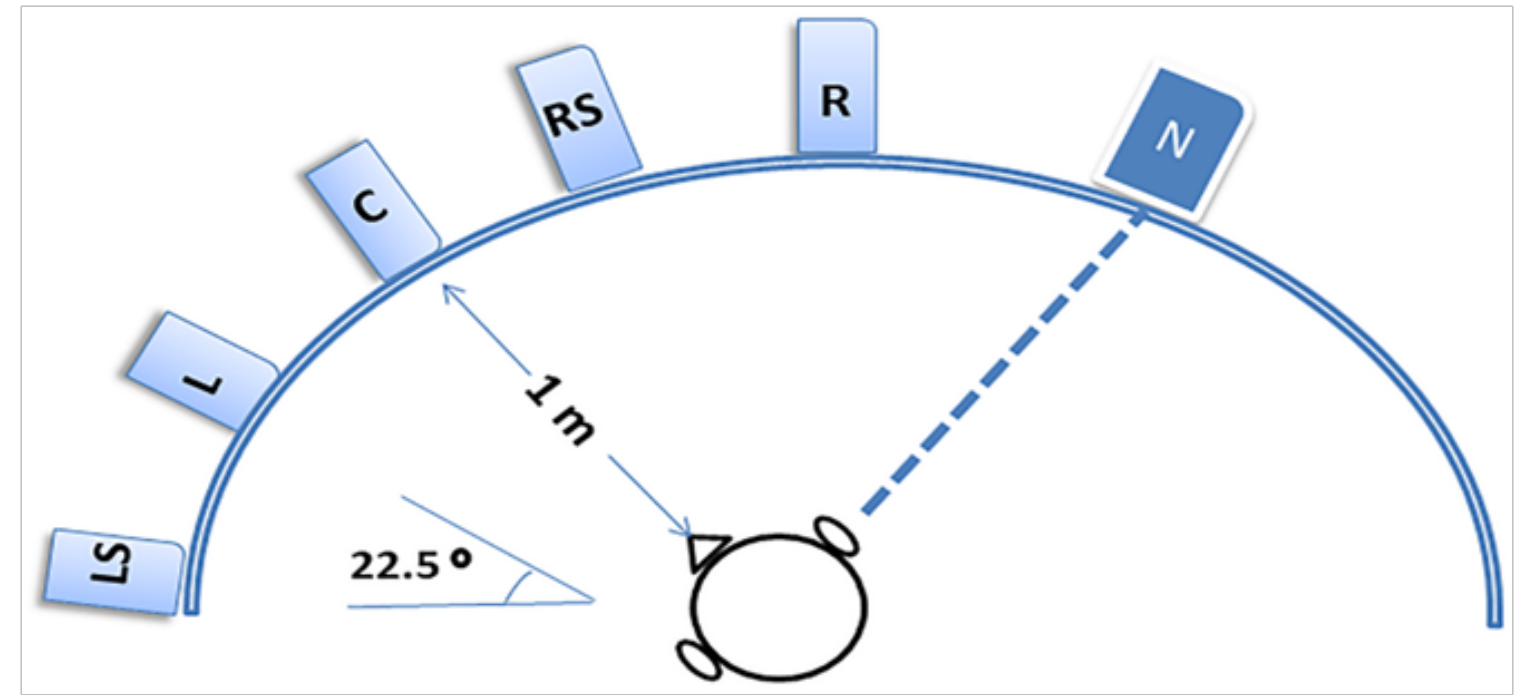

Fig. 1: Sound localization Test setting; speakers were positioned in a semicircle at 22.5 -degree intervals $1 \mathrm{~m}$ from the subject.

Each of the tonal stimuli $(250,4000 \mathrm{~Hz})$ was presented for $200 \mathrm{msec}$ while speech stimuli were presented for 1 sec. and $145 \mathrm{msec}$

The stimuli were presented among speakers randomly; each stimulus was presented 2 times from each speaker in each quarter.

The Background noise used was a broad-spectrum noise. Noise was presented in a signal to noise ratio $\mathrm{S} / \mathrm{N}=-10$.

\section{Data analysis}

The error score was calculated as 1 point for each 22.5-degree difference between the stimulus-presenting speaker and the speaker identified by the subject. The total number of points was then divided by the number sound localization trials performed. Data were analyzed using IBM SPSS version 20.0(Armonk, NY: IBM Corp). The Kolmogorov-Smirnov test was used to verify normality, and two-tailed $t$ tests were used to compare mean total error scores between the two groups.The Friedman test was used to compare the sound-localization ability based on the type of stimuli used. Qualitative data are presented as numbers with percentages, and quantitative data are presented as means with standard deviations or medians with ranges (minimum and maximum).

\section{RESULTS}

The tinnitus group included 20 patients with tinnitus. Two patients (10\%) were diagnosed with grade I tinnitus, five patients $(25 \%)$ with grade II tinnitus, 12 patients $(60 \%)$ with grade III tinnitus, and one patient (5\%) with grade IV tinnitus, according to the Dizziness Handicap Inventory. The mean (SD) age of the patient group was 33.30 (10.07) years. Fourteen subjects (70\%) were female, and six (30\%) were male.

The control group $(n=20)$ consisted of $14 \quad(70 \%)$ women and six $(30 \%)$ men with no history of tinnitus or any other neuro-otological problems. The mean (SD) age of the control group was 31.05 (15.51) years.

\section{Hearing test results}

Although not statistically significant, the tinnitus group showed mildly elevated thresholds at $6000 \mathrm{~Hz}$ and 8000 $\mathrm{Hz}$ than those of the control group.

Using the Friedman test, we found a significant difference between the localization ability in the quiet condition for pure-tone versus speech stimuli. Both groups showed the poorest localization ability for $4000-\mathrm{Hz}$ tones $0.31 \pm 0.16$, better localization ability for $250-\mathrm{Hz}$ tones $0.19 \pm 0.16$, and the best localization ability for speech stimuli $0.07 \pm 0.11 P<0.001$. For the noisy condition also a significant difference between the localization ability in the quiet condition for pure-tone versus speech stimuli. Both groups showed the poorest localization ability for $4000-\mathrm{Hz}$ tones $0.59 \pm 0.26$, better localization ability for $250-\mathrm{Hz}$ tones $0.46 \pm 0.21$, and the best localization ability for speech stimuli $0.33 \pm 0.20 P<0.001$. (Figure 2: Sound localization averages of error scores in both tinnitus and control group in quiet and noisy condition with three different stimuli).

Two-tailed $\mathrm{t}$ tests showed that the tinnitus group had significantly higher mean total error scores than the control group for all tested stimuli and under both testing conditions (Table 1).

There was no correlation between tinnitus pitch, loudness and TES nor DHI $(\mathrm{r}=0.26, p 90.05$ and $\mathrm{r}=0.08$, $P 90.0510 .6=P 0.12=\mathrm{r} 0.41=P 0.21=$ respectively $\mathrm{r}$ 


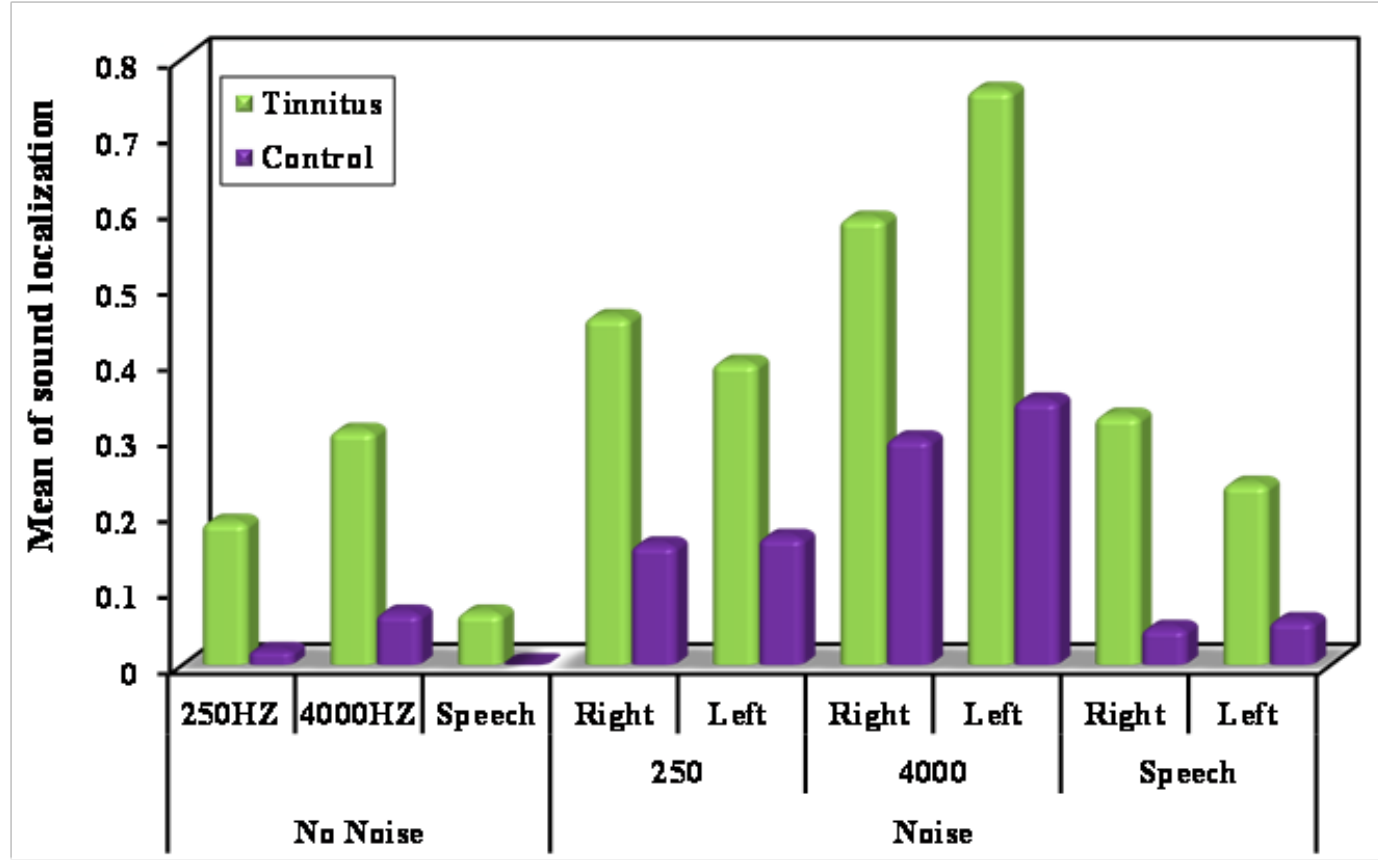

Fig. 2: Sound localization averages of error scores in both tinnitus and control group in quiet and noisy condition with three different stimuli.

Table 1: Comparison of all stimuli between tinnitus and control groups in both quiet and noisy conditions

\begin{tabular}{|c|c|c|c|c|c|}
\hline & \multicolumn{2}{|c|}{$\begin{array}{c}\text { Tinnitus group } \\
\mathrm{N}=20\end{array}$} & \multicolumn{2}{|c|}{$\begin{array}{c}\text { Control group } \\
\mathrm{N}=20\end{array}$} & \\
\hline & \multicolumn{2}{|c|}{ Mean error score } & \multicolumn{2}{|c|}{ Mean error score } & $P$ value \\
\hline \multicolumn{6}{|c|}{$250 \mathrm{~Hz}$} \\
\hline Quiet condition & \multicolumn{2}{|c|}{$0.19 \pm 0.16$} & \multicolumn{2}{|c|}{$0.02 \pm 0.04$} & $<0.001 *$ \\
\hline Noise toward the right ear & \multicolumn{2}{|c|}{$0.40 \pm 0.34$} & \multicolumn{2}{|c|}{$0.17 \pm 0.16$} & $0.008^{*}$ \\
\hline Noise toward the left ear & \multicolumn{2}{|c|}{$0.46 \pm 0.21$} & \multicolumn{2}{|c|}{$0.16 \pm 0.18$} & $<0.001^{*}$ \\
\hline$P$ value & Noise right $0.002 *$ & Noise left $0.012^{*}$ & Noise right $0.002^{*}$ & Noise left $0.003^{*}$ & \\
\hline \multicolumn{6}{|c|}{$4000 \mathrm{~Hz}$} \\
\hline Quiet condition & \multicolumn{2}{|c|}{$0.31 \pm 0.16$} & \multicolumn{2}{|c|}{$0.07 \pm 0.07$} & $<0.001 *$ \\
\hline Noise toward the right ear & \multicolumn{2}{|c|}{$0.76 \pm 0.25$} & \multicolumn{2}{|c|}{$0.35 \pm 0.27$} & $<0.001^{*}$ \\
\hline Noise toward the left ear & \multicolumn{2}{|c|}{$0.59 \pm 0.26$} & \multicolumn{2}{|c|}{$0.30 \pm 0.21$} & $<0.001^{*}$ \\
\hline$P$ value & Noise right $0.001^{*}$ & Noise left $<0.001 *$ & $\begin{array}{c}\text { Noise right } \\
<0.001^{*}\end{array}$ & Noise left $0.002^{*}$ & \\
\hline \multicolumn{6}{|c|}{ Speech } \\
\hline Quiet condition & \multicolumn{2}{|c|}{$0.07 \pm 0.11$} & \multicolumn{2}{|c|}{$0.0 \pm 0.0$} & $0.006^{*}$ \\
\hline Noise toward the right ear & \multicolumn{2}{|c|}{$0.24 \pm 0.20$} & \multicolumn{2}{|c|}{$0.06 \pm 0.12$} & $0.003 *$ \\
\hline Noise toward the left ear & \multicolumn{2}{|c|}{$0.33 \pm 0.20$} & \multicolumn{2}{|c|}{$0.05 \pm 0.10$} & $<0.001 *$ \\
\hline$P$ value & Noise right $0.001^{*}$ & Noise left $0.002 *$ & Noise right 0.066 & Noise left 0.066 & \\
\hline
\end{tabular}

$P$ values for comparing the quiet and noisy environment results for each group.

*: Significant at $P \leq 0.05$ 


\section{DISCUSSION}

Results of the current study revealed that tinnitus patients with normal hearing had a decreased ability to localize sounds in both acoustically favorable and unfavorable environments, compared with control subjects. Furthermore, the localization ability was worse for highfrequency stimuli $(4000 \mathrm{~Hz})$ than for low-frequency stimuli $(250 \mathrm{~Hz})$ in both tested groups, which may have resulted from the effects of tinnitus on interaural intensity differences ${ }^{[10]}$. Specifically, a previous study has shown that tinnitus interferes more significantly with localization when sounds originate from the same side as the tinnitus $^{[11]}$. This principle supports our observation that there was a significant difference between speech localization error scores in noisy versus quiet conditions in the tinnitus group only.

The exact mechanism leading to differences in auditory task performance between tinnitus patients and subjects with normal audiograms under noisy conditions remains unclear ${ }^{[11]}$. However, one possible cause could be a tinnitusassociated decrease in the quantity of afferent auditory nerve filaments. This loss, known as deafferentation, has been described as "hidden hearing loss" because it is not possible to identify it using standard audiometric methods ${ }^{[12]}$. Specifically, one theory is that auditory nerve deafferentation affects the medial olivocochlear system, which is responsible for sound localization, especially under noisy conditions ${ }^{[13]}$. Deafferentation leads to a dyssynchrony of the auditory fibers, thus disabling the sound-localization ability, particularly in noisy environments ${ }^{[14]}$. Another explanation for the relatively poor ability of tinnitus patients to localize speech stimuli under noisy conditions may be the importance of extended high frequencies $(8000-16000 \mathrm{~Hz})$ for speech localization $^{[15]}$

\section{CONCLUSION}

The participants with tinnitus in our study exhibited a diminished ability to localize sound compared with sexmatched controls, and their performance worsened for speech localization under noisy conditions. Further studies with larger sample sizes and multiple demographic traits are necessary to confirm these results.

\section{CONFLICT OF INTEREST}

There are no conflicts of interest.

\section{REFERENCES}

1. Onishi ET, Coelho CCB, Oiticica J, Figueiredo RR, Guimarães RCC, Sanchez TG, et al. Tinnitus and sound intolerance: evidence and experience of a Brazilian group. Braz J Otorhinolaryngol. 2018;84:135-49.
2. Sanchez TG, de Medeiros IRT, Levy CPD, Ramalho JDO, Bento RF. Tinnitus in normally hearing patients: clinical aspects and repercussions. Braz J Otorhinolaryngol. 2005;71:427-31.

3. Galazyuk AV, Wenstrup JJ, Hamid MA. Tinnitus and underlying brain mechanisms. CurrOpinOtolaryngol Head Neck Surg. 2012;20:409-15.

4. Moring J, Bowen A, Thomas J, Bira L. The emotional and functional impact of the type of tinnitus sensation. J ClinPsychol Med Settings. 2016;23:310-8.

5. Haider HF, Bojić T, Ribeiro SF, Paço J, Hall DA, Szczepek AJ. Pathophysiology of subjective tinnitus: triggers and maintenance. Front Neurosci. 2018 27;12:866.

6. An YH, Jin SY, Yoon SW, Shim HJ. The effects of unilateral tinnitus on auditory temporal resolution: gaps-in-noise performance. Korean J Audiol. 2014;18:119-25.

7. Wang Y, Zhang JN, Hu W, Li JJ, Zhou JX, Zhang JP, et al. The characteristics of cognitive impairment in subjective chronic tinnitus. Brain Behav. 2018;8:e00918.

8. Middlebrooks JC. Sound localization. In: Aminoff MJ, Boller F, Swaab DF, eds. The Human Auditory System Fundamental Organization and Clinical Disorders. Elsevier; 2015: 99-116.

9. Newman CW, Jacobson GP, Spitzer JB. Development of the Tinnitus Handicap Inventory. Arch Otolaryngol Head Neck Surg. 1996;122:143-8.

10. An YH, Hyung LL, Yoon SW, Jin SY, Shim HJ. Does tinnitus affect the sound localization ability? OtolNeurotol. 2012;33:692-8.

11. Weisz N, Hartmann T, Dohrmann K, Schlee W, Norena A. High-frequency tinnitus without hearing loss does not mean absence of deafferentation. Hear Res. 2006;222:108-14.

12. Plack CJ, Barker D, Prendergast G. Perceptual consequences of "hidden" hearing loss. Trends Hear. 2014;18:2331216514550621.

13. Breuel MLF, Sanchez TG, Bento RF. Vias auditivas eferentes e seu papel no sistema auditivo. ArqOtorrinolaringol. 2001;5:62-7.

14. Guinan JJ. Olivocochlearefferents: anatomy, physiology, function, and the measurement of efferent effects in humans. Ear Hear. 2006;27:589-607.

15. Best V, Carlile S, Jin C, van Schaik A. The role of high frequencies in speech localization. J AcoustSoc Am. 2005; 118:353-63. 